\title{
THE ROTATION MEASURE OF BACKGROUND RADIO SOURCES SEEN THROUGH THE SUPERNOVA REMNANT OA184 (G166.2+2.5)
}

\author{
K.-T. Kim and P. P. Kronberg, University of Toronto, Canada \\ T. L. Landecker, Dominion Radio Astrophysical Observatory, Penticton, B.C., \\ Canada
}

\begin{abstract}
Radio sources in the field of the extended SNR OA184 (G166.2+2.5) have been studied to determine the excess rotation measure (RM) arising from the SNR. Of a total of 32 radio sources observed with the VLA in the C configuration, eight are found to be polarized above $7 \sigma$. The sources seen through the SNR show significantly high RM in comparison to background sources. The excess RM due to the Faraday active plasma in the SNR is estimated to be $150 \pm 20 \mathrm{rad} \mathrm{m}^{-2}$, which corresponds to $\left\langle n_{e} B_{-6}\right\rangle_{\mathrm{rim}}=(30 \pm 5) L_{10 p c}^{-1}$. The sign of RMs of the sources within an area of about $2^{\circ} \times 2^{\circ}$ centred on the SNR shows a systematic longitudinal polarity change on either side of $l \approx 166^{\circ} .2$. Although a larger sample is needed to justify this, we tentatively interpret this "flip" as due to the reversal of an irregular component of the galactic magnetic field on a scale of order $100 \mathrm{pc}$.
\end{abstract}

\section{INTRODUCTION:}

Polarization measurement using background sources as probes is known to be an efficient technique for investigation of the magnetic field structure of the intervening medium if a sufficient number of "Faraday" probes can be obtained. This technique has been used for galactic objects, such as the Gum nebula (Vallée and Bignell 1983), the Monogem region (MacLeod et al. 1984) and notably for the whole Galaxy (see Simard-Normandin and Kronberg 1980 and references therein) by examining the spatial correlations of the Faraday rotations of a large number of extragalactic sources. This technique should be applicable to supernova remnants (SNRs). Unfortunately SNRs are small; the average angular size of 125 SNRs listed by Milne (1979) is $35^{\prime} \pm 5^{\prime}$. Only a small number of background sources are seen through most SNRs and therefore this type of study has been previously applied only to very extended "bubbles".

The large angular extent of the SNR OA184 (G166.2+2.5; 76' in diameter) makes it well suited to a "Faraday Probe" experiment using polarized background sources. A previous DRAO map has revealed 32 background radio sources whose flux density at $1.4 \mathrm{GHz}$ exceeds $25 \mathrm{mJy}$ in the $2^{\circ} \times$ $2^{\circ}$ area centred on OA184 (Routledge, Landecker and Vaneldik 1986, RLV). Furthermore, OA184 lies towards the galactic anticentre and is above the galactic plane. This makes OA184 an ideal SNR to probe for rotation measure (RM) variations, since the general interstellar medium (ISM) component of RM is small at this longitude (cf. Simard-Normandin and Kronberg 1980), and is therefore less likely to overwhelm the contribution from the SNR shell itself. This paper describes the results of an attempt to study the magnetic field strength in the SNR OA184 by observing the excess of Faraday rotation measures of background radio sources in the field.

From a priori estimates of physical conditions in OA184 $\left(n_{e} \sim 1, B_{-6} \sim 1, \operatorname{size}(L) \approx 100 p c\right.$ [RLV, revised diameter, Landecker, private communication 1987]), the RM produced in the SNR might easily be of the order of $100 \mathrm{rad} \mathrm{m} \mathrm{m}^{-2}$. ( $B_{-6}$ is the magnetic field strength in microgauss, $n_{e}$ is the electron density in $\mathrm{cm}^{-3}$ and $L$ is the pathlength). This estimate of RM is well supported by the results given in Bignell and Vallée (1983). 
Figure 1

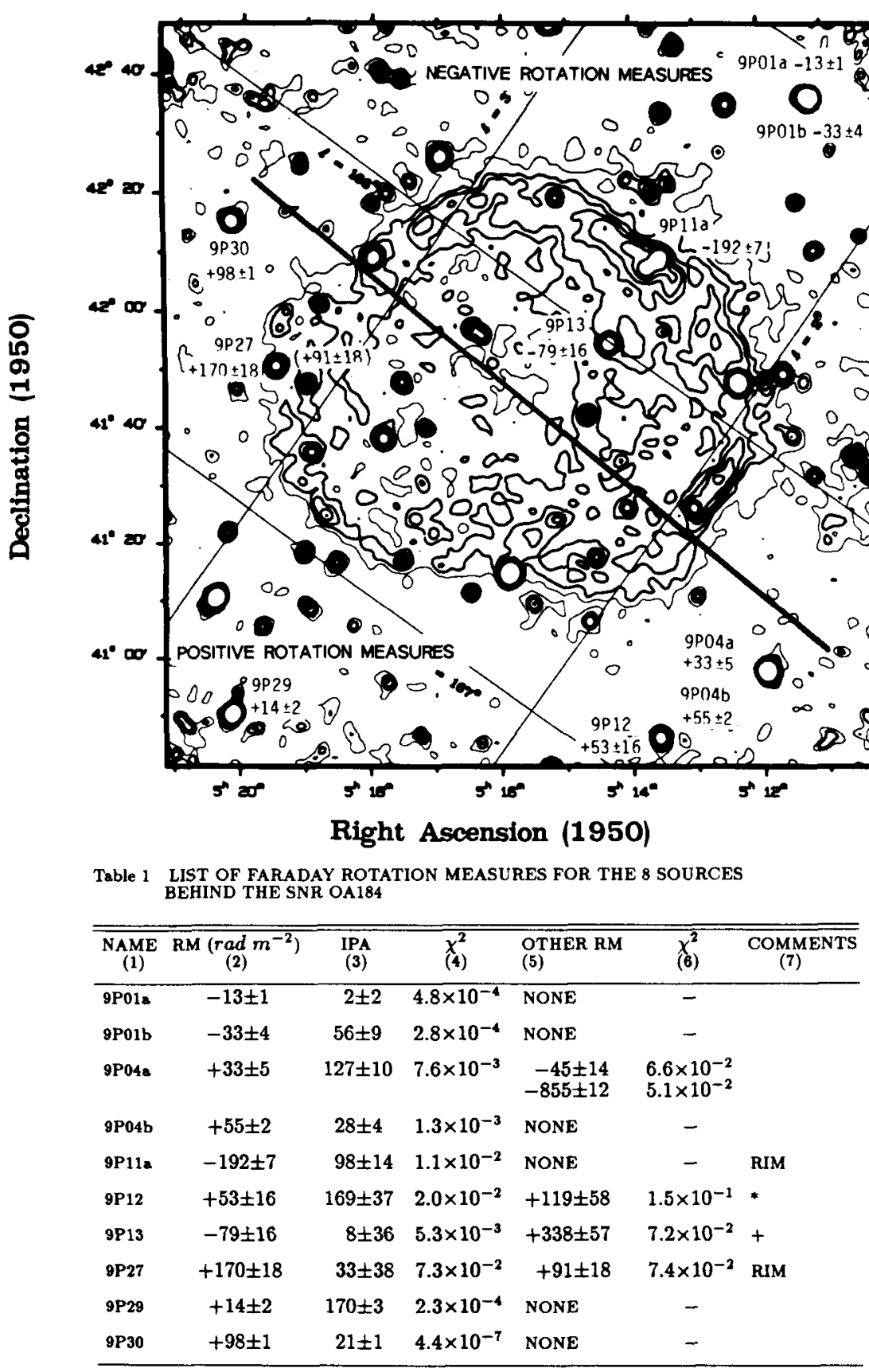

Note: (1) Narne of the source, (2),(3), Accepted value of Faraday Rotation Measure and Intrinsic Polarization Position Angle in degrees, (4) chi-square values of lesst-squarea fit, (5) Other acceptable RMs, (6) chi-square values for RMs in column (5), (7) Comments; RIM = source is superimposed on the rim of the SNR, + Source seen through the SNR, * Ambiguous RM 


\section{OBSERVATIONS AND RESULTS:}

Observations were made with the VLA* in the C configuration at $1385,1465,1515,1665,4835$ and $4885 \mathrm{MHz}$. In Table 1, we list the RMs measured for the 8 sources in the field of SNR OA184 which had detectable polarization. These sources are marked on the map of OA184 in Figure 1, and the measured RMs are indicated.

An intriguing result evident in Figure 1 is that the signs of the RMs are opposite on either side of a line drawn at $l \approx 166.2^{\circ}$. This separation of the signs of the RMs could occur by chance, and a series of computer simulations was therefore performed to investigate this. The probability that 8 sources with random RMs should by chance fall into two groups of 5 and 3 separated by a straight line (as shown in Table 1 and Figure 1) was calculated in the Monte Carlo manner. It proved to be $P(5: 3)=19.8 \pm 1.4 \%$ after several thousand simulations. However, the observed distribution has greater significance than this because the double sources 9P01 and 9P04 both have two components showing RM of the same sign. Therefore a further probability $p_{p}^{2}$ was applied, where $p_{p}=\frac{1}{2}$ is the probability that the two components of a double source have the same sign of RM. Then the probability of chance occurrence of the observed distribution of RMs is

$$
P_{2 D}(5: 3)=P(5: 3) \times p_{p}^{2}=5 \pm 2 \% .
$$

This probability is by coincidence about the same as $P(6: 4)=3.6 \pm 0.6 \%$.

For the cases of equal numbers of sources of each sign, the probabilities $P(2: 2), P(3: 3)$, $P(4: 4), P(5: 5)$, and $P(6: 6)$ are estimated to be $81 \pm 3 \%, 40 \pm 2 \%, 16.5 \pm 1.3 \%, 4.0 \pm 0.6 \%$, and $2.0 \pm 0.4 \%$. This implies that the significance of the suggested polarity flip would be firmly tested with a small number of additional sources in the field. It is unfortunate that of 32 sources observed, only 8 showed measurable polarization.

\section{DISCUSSION:}

The average RM of the background sources outside the SNR is $\langle|R M|>\approx 40 \pm 13 \mathrm{rad} \mathrm{m}-2$ (for 7 sources), whereas that of the inside sample (for 3 sources) is $\left\langle|R M|>\approx 150 \pm 30 \mathrm{rad} \mathrm{m}^{-2}\right.$. Taking the mean separately according to the sign of the RM, we have $+50 \pm 15$ and $-23 \pm 10$ rad $m^{-2}$ for each side of the SNR. Note that these are small number statistics.

It is particularly noteworthy that the two polarized sources lying in the radio continuum "rim" have large rotation measures. These are 9P11a $\left(-192 \mathrm{rad} \mathrm{m}^{-2}\right)$ and 9 P27 $\left(+170 \mathrm{rad} \mathrm{m}^{-2}\right)$. This reinforces the sense of the statistics above, and suggests that we are detecting an excess RM due to the SNR.

The excess amount of RM from the rim of the SNR can be estimated as follows. Taking the mean of the two RMs, -192 and $170 \mathrm{rad} \mathrm{m^{-2 }}$, of the sources in the rim, and assuming that the pathlength intercepting the SNR is less than $10 \%$ of the diameter of OA184, that is about $10 \mathrm{pc}$, we have

$$
n_{e} B_{-6}=18 \pm 2 L_{10 p c}^{-1}(\cos \theta)^{-1},
$$

where $L_{10 p c}$ is the pathlength in units of $10 p c$ and $\theta$ is the angle between the line of sight and the magnetic field. By taking an average RM of the sources seen outside the SNR, the mean intrinsic $\mathrm{RM}$ of the background sources, $\langle|R M|\rangle_{\text {inc }}$, is estimated to be $36 \mathrm{rad} \mathrm{m^{-1 }}$ and this amount is subtracted to estimate the net RM of the SNR. For an old SNR like OA184 the magnetic field configuration is dominated by a circumferential component (Milne 1987). Hence, we can assume that $\theta$ lies in a plane tangent to the rim of the SNR. As a good approximation, where the line of sight passes through the rim of the SNR, we can estimate that $<\cos \theta>=0.54$ and we expect:

$$
<n_{e} B_{-6}>_{\text {rim }} \approx(30 \pm 5) L_{10 p c}^{-1} .
$$

* The Very Large Array of the National Radio Astronomy Observatory is operated by Associated Universities Inc. under contract to the National Science Foundation. 
This amount of $n_{e} B_{-6}$ is consistent with the value inferred from shock wave amplification of $n_{e} B$ in the ambient galactic medium.

Another way of estimating the magnetic field of the SNR is to use the equipartition argument. Using the flux density at $1.4 \mathrm{GHz}$ of $9.0 \mathrm{Jy}$ and $\alpha=-0.54$ (Routledge et al. 1987; Milne 1979), we have

$$
B_{e q}=4.1\left(\frac{1+k}{\psi}\right)^{\frac{2}{7}} \text { microgauss, }
$$

where $\psi$ is the volume filling factor, $k$ is the proton/electron energy density and $100 \mathrm{pc}$ is used as the diameter of OA184. The edge-brightened morphology of OA184 suggests that most of the total radio flux is likely to originate from a thin shell where the interactions of the SNR with the ISM are intense. Assuming the thickness of the shell is of the order of $10 \%$ of the diameter of OA184, then the best estimate on the term in parentheses in eq $(4),\left(\frac{1+k}{\psi}\right)^{\frac{7}{7}}$, which is relatively insensitive to $k$, is about $4-6$ for $k=1-100$. Substituting this estimate into eqs (4)and (3), then gives

$$
\begin{gathered}
B_{e q} \approx 16 \pm 8 \text { microgauss, } \\
<n_{e}>_{\operatorname{rim}} \approx(1.9 \pm 1.2) L_{10 p c}^{-1} .
\end{gathered}
$$

These estimates on $B$ and $n_{e}$ are in good agreement with those from other studies (e.g., Milne 1987).

The longitudinal gradient in RM across OA184 is estimated to be about $300 \mathrm{rad} \mathrm{m} /$ degree. It is interesting to compare this result with those from two previously studied SNRs. Both appear to show a polarity flip in RM; they are 1209-51/52 and Puppis A (Dickel and Milne 1976). The polarity flip appears not to be associated with the SNR itself, since background sources located well away from the centroid of the SNR also show this trend. Instead the SNR, as it expands, amplifies the ambient galactic magnetic field and enhances the contrast in magnetic field polarity already existing in the galactic field.

The location of OA184, especially the longitude, is of particular interest. The galactic longitude $l \approx 165^{\circ}$ is perpendicular to the Orion and Perseus arms (Simard-Normandin and Kronberg 1980; Vallée and Bignell 1983), and the galactic RM arising from the regular component of galactic magnetic field threading the spiral arms should be small. Hence the RM distribution of the sources in the field of OA184 might reflect the spatial coherence of the random field component of the general interstellar magnetic field. Based on the data presented here, the scale size of the magnetic field reversal should be of the order of a degree, about $100 \mathrm{pc}$ in linear dimension, to be consistent with the polarity flip shown in the map. However, of course, more data are needed to better specify the scale size and strength of the random magnetic field reversals.

\section{REFERENCES:}

Dickel, J. R., and Milne, D. K. 1976, Aust. J. Phys., 29, 435.

Macleod, J. M., Vallée, J. P., and Broten, N. W. 1984, Astron. Astrophys. Suppl. Ser. 56, 283.

Milne, D. K., 1979, Aust. J. Phys., 32, 83.

Milne, D. K. 1987, Preprint.

Routledge, D., Landecker, T. L., and Vaneldik, J. F., 1986, M.N.R.A.S., 221, 809. [RLV]

Simard-Normandin, M., and Kronberg, P. P., 1980, Ap. J., 242, 74.

Simard-Normandin, M. 1980, Ph.D. Thesis University of Toronto.

Vallée, J. P. and Bignell, R. C., 1983, Ap. J.,272, 131. 